psychopraxis. neuropraxis $2022 \cdot 25: 45-48$ https://doi.org/10.1007/s00739-021-00774-5 Angenommen: 7. Dezember 202 Online publiziert: 5 . Januar 2022

(c) Der/die Autor(en) 2022

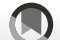

\section{Steinmair ${ }^{1,2,3} \cdot$ A. Ronge-Toloraya $^{4} \cdot$ H. Löffler-Stastka'}

' Klinik für Psychoanalyse und Psychotherapie, Medizinische Universität Wien, Wien, Österreich

${ }^{2}$ Karl Landsteiner Privatuniversität für Gesundheitswissenschaften, Krems, Österreich

${ }^{3}$ Klinische Abteilung für Augenheilkunde, Universitätsklinikum St. Pölten, St. Pölten, Österreich

${ }^{4}$ Unit für Postgraduelle Aus- und Weiterbildung, Medizinische Universität Wien, Wien, Österreich

\title{
Veränderungen der Kontextfaktoren und deren Auswirkungen auf die Arzt- Patient-Beziehung
}

\section{Einleitung}

Die therapeutische Beziehung ist als Wirkfaktor für den Therapieerfolg in den Fokus der Aufmerksamkeit gerückt. Wie aber wirkt sich der Kontext einer Behandlung auf die Gestaltung dieser Beziehung aus?

\section{Gesellschaftliche Perspektiven}

Der gesellschaftliche Rahmen menschlichen Agierens ist ein kontinuierlicher Prozess, der von individuellen Handlungen getragen eigene Regeln und Regelmäßigkeiten zeitigt, die wiederum auf das Handeln der Akteure einwirken. Ein beschleunigter gesellschaftlicher Wandel beinhaltet Begriffe wie Globalisierung, Produktivitätssteigerung, Technisierung/Digitalisierung [1].

An Demokratisierungsprozesse knüpften politische Kämpfe an, um Freiheitsrechte einzufordern, aus welchen letztlich beispielsweise die PatientInnenEmpowerment-Bewegung hervorgegangen ist [2]. Paternalistische Sichtweisen innerhalb der Medizin wurden kritisiert und Zuschreibungen dem/r PatientIn als hilfsbedürftigem/r AkteurIn zurückgewiesen. Forderungen nach mehr Autonomie und einem Arzt-Patient-Verhältnis auf Augenhöhe wurden artikuliert [3]. Die Dominanz der ExpertInnen wird infrage gestellt, die Kompetenzen auf Seite der PatientInnen werden betont und wissenschaftliche Belege für dia- gnostisches und therapeutisches Tun gefordert [4]

Insgesamt wird eine Verschiebung vom Autoritäts- und Herrschaftsverhältnis zum Vertragsverhältnis konstatiert. Der Anspruch verändert sich: Aus dem Arzt-Patient-Verhältnis wird das ArztKunden-Verhältnis [5]. Diese Perspektive birgt jedoch die Gefahr, die „Rationierung von Gesundheitsleistungen als ,Marktgeschehen “" zu interpretieren [6]. Krankenbehandlung bleibt - trotz Kritik am Paternalismus - auf Wissensbestände und institutionalisierte Kompetenzen der BehandlerInnen angewiesen [7].

Gleichzeitig bilden PatientInnen keine passiven „Objekte“, sondern aktive Elemente ihrer eigenen Krankenbehandlung, treten als mehr oder weniger selbstbewusste Ko-ProduzentInnen [8] von Gesundheit auf; als „active patients“ [9], „informed patients“ [10] oder „patients as experts" [11], deren Mitarbeit auch zunehmend eingefordert wird.

\section{》) Die gelungene partner- schaftliche Beteiligung der PatientInnen ist voraussetzungs- voll}

Insgesamt kann ein Wandel hin zu partizipativen Entscheidungsfindungen beobachtet werden, ohne dass paternalistische Elemente verschwinden. Vielmehr besteht beides fort als Pole eines
Kontinuums. Sie werden je nach Behandlungssituation, Ausstattung der beteiligten Akteure, Art und Schweregrad der Erkrankung mehr oder weniger relevant und situativ aktiviert. In Kürze bleibt die Einsicht: Es gibt nicht „das“ Arzt-Patient-Verhältnis, sondern eine Vielzahl von Verhältnissen und Begegnungen im zunehmend fragmentierten Behandlungsnetzwerk [12]. „Ein einfaches Rezept für eine ,gute Beziehung ist daher kaum möglich" [13]. Die gelungene partnerschaftliche Beteiligung der PatientInnen ist voraussetzungsvoll und fordert ein hohes Maß an affektkognitiver und körperlicher Leistungsfähigkeit trotz Erkrankung, aber z. B. auch ausreichende „Health Literacy“" $[14,15]$.

Trialogprozesse, die Angehörigenbewegungen inkludieren, sind noch nicht ausreichend als Elemente der Alltagskultur etabliert und Probleme sind hinsichtlich der Einführung eines entmetapsychologisierten Normierungssystems mit autonomen Technisierungsfolgen ohne systematische, prozesshafte Expertenprüfung angesprochen.

Zum Mangel an validen, nur im Sinnzusammenhang interpretierbaren Daten tritt die Herausforderung, den Informations-Overload [16] zu be- und verarbeiten. Aus verfügbaren Informationen belastbares Wissen zu integrieren, erfordert eine gelungene HealthLiteracy-Strategie, die wiederum auf Basis einer guten therapeutischen Beziehung - Adhärenz - entstehen kann. 
Die Generierung diffuser Ängste durch kurze Fehlschlüsse oder Fake News und die paranoiden Exzesse aufgrund fehlender Expertenbewertung führen $\mathrm{zu}$ kaum zu kontrollierenden, hoch emotionalisierten Fanatisierungsprozessen, die faktenbasierte reflexive Mentalisierungsprozesse nötig machen durch vertrauenswürdige ExpertInnen in den globalen Informationscommunities.

Zweifellos hatte die COVID-19-Pandemie einen ungünstigen Einfluss auf die psychische Gesundheit [17-19], während Therapien durch Kontaktbeschränkungen erschwert waren. Maßnahmen zur Eindämmung der Pandemie (Ausgangsbeschränkungen, Quarantäne, Kontaktverfolgung) führten zu zahlreichen Einschränkungen in der individuellen Selbstbestimmung, zum Schutz von Leben unter dem Vorzeichen der Solidarität, zur Sicherung der Freiheit ohne Gefährdung der Gemeinschaft durch den Einzelnen [20, 21]. Krisenbedingt wurde das Online-Setting zur neuen Normalität. Die bisherige Studienlage zur Güte der therapeutischen Beziehung unter diesen Bedingungen zeigte, dass die Arbeitsallianz im VideotelefonieSetting dem Face-to-face-Setting unterlegen war [22]. Longitudinale Studien versprechen allerdings einen besseren Einblick in die Folgen der Pandemie [19] und Folgen von Veränderungen des therapeutischen Settings als die bislang überwiegend durchgeführten Querschnittstudien, welche sich vor allem auf Selbstberichte (von betroffenen PatientInnen/TherapeutInnen) beschränken. Eine notwendige Adaptation therapeutischer Techniken und gleichzeitig das Vermeiden von Grenzverletzungen im Online-Setting können eine Herausforderung darstellen [23]. Lockdowns waren in Selbstberichten mit höherer Aggressionsbereitschaft, Ärger und Feindseligkeit in der Bevölkerung assoziiert, welche sich physisch und verbal äußerten [24]. Diese Entwicklung passt zur „FrustrationAggression“-Hypothese von Berkowitz [25]: Wird die Erreichung eines gewünschten Zieles (Autonomie, SelbstEffektivität, Beziehung/Bindung etc.) vereitelt, ist dies ausreichend für das Entstehen negativer Affektivität, welche dann eine Aggressionsneigung bedingt
[24, 26]. Selbstberichtete Aggressivität muss sich nicht in entsprechendem Verhalten äußern, allerdings war während der Lockdowns auch Gewalt [27-30] dokumentiert worden. Die Empfehlung an TherapeutInnen, PatientInnen hinsichtlich Zeichen erhöhter Aggressivität $\mathrm{zu}$ evaluieren, gilt besonders während Lockdowns [24].

\section{Fallvignette}

„Ich habe diese Ordination gekauft. Die Ordination gehört mir, Sie gehören mir. " Frau $\mathrm{T}$ ist eine unscheinbare Erscheinung. Auf dem Sessel in sich versunken und in schmuddelige Kleidung gehüllt, flüstert sie diese Worte wie $\mathrm{zu}$ sich selbst, zupft eine Haarsträhne vor das schmale Gesicht - wie einen Vorhang, zum Dahinterverschwinden. Frau T, 25jährig und seit 2 Jahren arbeitslos, hat ihre Lehre zur Verkäuferin abgebrochen; sie lebt seither sozial zurückgezogen aufgrund von paranoiden Ängsten. Als der Behandler nach dem Befinden von Frau T fragt, fängt diese plötzlich an zu schreien, wobei sie perseveriert, diese Ordination gekauft zu haben und insgesamt für eine große Aufgabe berufen zu sein. Ohne ersichtlichen Grund scheint sie sich kurz danach wieder beruhigt $\mathrm{zu}$ haben und gibt nun Anweisungen an den Behandler, einen Beschwerdebrief $\mathrm{zu}$ schreiben, da man versuche, sie zu vergiften. Als der Behandler dieser Anordnung nicht unmittelbar nachkommt, betastet Frau T das eigene Gesicht. „Ich verliere mein Gesicht. Mein Gesicht ist weg. "Frau T rutscht nervös auf dem Sessel hin und her und scheint zu überlegen, die Stirn ist in tiefe Falten gerunzelt, die Augen dem Blick des Behandlers stets ausweichend. Weiterhin sei sie davon überzeugt, verfolgt und vergiftet worden zu sein, „gesichtsauflösende Medikamen$t e$ " seien die Ursache, sie selbst habe sie sich verschrieben, beschließt sie den Dialog.

\section{Diskussion}

$\mathrm{Zu}$ Beginn der Intervention steht die Etablierung einer therapeutischen Beziehung, welche empathisches sich Einfühlen und Mentalisieren erfordert. Wie psychopraxis. neuropraxis $2022 \cdot 25: 45-48$ https://doi.org/10.1007/s00739-021-00774-5 (c) Der/die Autor(en) 2022

\section{Steinmair - A. Ronge-Toloraya • H. Löffler-Stastka \\ Veränderungen der Kontextfaktoren und deren Auswirkungen auf die Arzt- Patient-Beziehung}

\section{Zusammenfassung}

Therapeutisches Wirken wird durch begünstigende Kontextfaktoren erleichtert, wobei therapeutische Interventionen gleichzeitig Anregung sein können, Kontextfaktoren zu verändern. Kommunikation und Therapie werden durch eine gute therapeutische Beziehung erst ermöglicht, vor allem unter erschwerten inneren und äußeren Bedingungen.

\section{Schlüsselwörter}

Beziehung - Empathische Fürsorge .

Autonomie · Aggression · Reflexion

\section{Changes in Contextual Factors and Their Effects on the Doctor-Patient Relationship}

\section{Abstract}

Therapeutic action is facilitated by favourable contextual factors whereby therapeutic interventions can also be an incentive to change contextual factors. Communication and therapy are only made possible by a good patient-therapist relationship, especially under difficult internal and external conditions.

\section{Keywords}

Attachment · Empathic Care · Autonomy . Aggression · Reflection

herausfordernd dies sein kann, wird besonders in dieser Begegnung mit einer Patientin sichtbar, welche externalisierend, projektiv-identifizierend und mittels Allmachtfantasien ihre Affekte reguliert. Affektives Containment und strukturierende Interventionen, welche Sicherheit und (Selbst-)Kontrolle vermitteln, sind nun notwendig [31, 32]. Den dominanten Affekt zu adressieren, stellt eine weitere effektive Intervention dar [33]. Der analytikerzentrierten [34] Intervention [35] im Hier und Jetzt (der therapeutischen Beziehung) kommt eine 
entscheidende Rolle zu. Die Begegnung von Frau $\mathrm{T}$ mit dem Behandler fand nicht im Videotelefonie-Setting statt.

》) Strukturierende Interventionen, die Sicherheit und (Selbst-)Kontrolle vermitteln, sind notwendig

Unabhängig vom Kontext, sind Behandler allerdings gefordert, die negativen Affekte im Jetzt der therapeutischen Beziehung zu „containen“, um die weitere therapeutische Arbeit zu ermöglichen. Erst auf Basis einer stabilen therapeutischen Beziehung ist das notwendige Durcharbeiten negativer Spitzenaffekte möglich [36].

Eine besondere Herausforderung stellt der Umgang mit Aggressivität dar, sei sie passiv/unterschwellig oder offen geäußert. Im Fall der Eskalation von Gewalt, der Selbst- oder Fremdgefährdung kommt der/die FachärztIn für Psychiatrie in die Situation, entsprechend den Kriterien des Unterbringungsgesetzes (UBG) paternalistisch für Patienten zu entscheiden und ihre Freiheit zu ihrem eigenen Wohl zu beschneiden. Als Gründe für Aggression im Gesundheitswesen zeigen sich auf Patientenseite ein hohes inneres Spannungsniveau und Aggressionspotenzial, mangelnde Einsicht und die Wahrnehmung von Maßnahmen als Gewalt anstatt Behandlung, eine eingeschränkte Kommunikationsmöglichkeit bei gleichzeitig hohem Erwartungsdruck [37]. Wird ein Verlust an Autonomie, Kontrolle, Ungerechtigkeit oder Hilflosigkeit erlebt, nimmt das Aggressionspotenzial zu. Erlittene Kränkungen und Demütigungen, Bestrafungen, die Verarbeitung von Trauer/Verlust oder Minderwertigkeitsgefühlen tragen zu Ärger, Gewalt und Aggression bei. BehandlerInnen sind aufgefordert, erste Anzeichen von Gewalt wahrzunehmen, Ruhe zu bewahren und die eigene Sicherheit zu beachten, wobei Körpersprache beobachtet und Blickkontakt hergestellt werden sollte [38]. Weder selbst $\mathrm{zu}$ provozieren noch sich provozieren $\mathrm{zu}$ lassen und eine wertschätzende Haltung zu bewahren, um Bedürfnisse und Ge- fühle des Gegenübers wahrnehmen und herausarbeiten zu können, bedarf einer ausreichenden Kommunikations- und Deeskalationskompetenz.

\section{》) Kommunikationskompetenz, Mentalisierungsfähigkeit und Arbeitsumfeld wirken zusammen}

Aggressive Kommunikation kann als Beziehungsversuch des/r PatientIn wahrgenommen werden, welcher in Ermangelung anderer Umgangsweisen mit Ärger und Wut in Erscheinung tritt. Neben Kommunikationskompetenz und Mentalisierungsfähigkeit trägt das Arbeitsumfeld dazu bei, ob Menschen in Gesundheitsberufen ihre volle Kompetenz in der Interaktion mit aggressiven PatientInnen entfalten können. Dauerfrustration, chronischer Stress, zahlreiche und hohe Spitzenstressoren tragen dazu bei, dass Empathie, Toleranz, Differenzierungsfähigkeit und soziale Kompetenzen schwinden können [37]. Neben dem Schaffen entsprechender Arbeitsbedingungen wirkt sich eine Stärkung der Mentalisierungsfähigkeit in stressreichen, emotionalisierten Situationen positiv aus.

Psychotherapeutische Interventionen zur Verbesserung von Schwierigkeiten im sozialen Umgang fokussieren zunehmend auf eine Verbesserung der Emotionsregulation und der kognitiven Fähigkeiten, welche für Anhedonie und Apathie relevant sind (e.g. Positive Emotions Program for Schizophrenia (PEPS) [39]). Die Güte der Emotionswahrnehmung, die kognitive Leistung einschließlich der sozialen Fähigkeiten sowie die klinische Symptomatik sind signifikante Prädiktoren der Mentalisierungsfähigkeit schizophrener Patienten [40]. Kontextfaktoren sind für die therapeutische Allianz relevant [41].

\section{Fazit für die Praxis}

\section{- Die Beziehung zwischen Ärztln und Patientln findet im Spannungsfeld zwischen Ethik der Fürsorge und Ethik der Autonomie statt. \\ - Professionelles Deeskalationsmana- gement ist im Umgang mit Gewalt}

und Aggression frühzeitig und zielgerichtet indiziert.

- Ohne Basis einer guten Bindung ist Kommunikation erschwert. Vor allem indizierte psychotherapeutische Interventionen lassen sich ohne etablierte Beziehung nicht adäquat einsetzen.

- Das Setting kann Therapieerfolge fördern oder erschweren.

\section{Korrespondenzadresse}

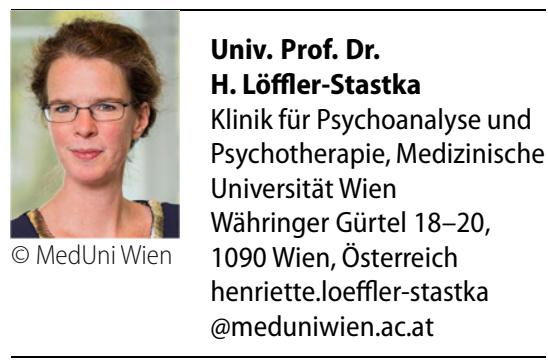

Funding. Open access funding provided by Medical University of Vienna.

\section{Einhaltung ethischer Richtlinien}

Interessenkonflikt. D. Steinmair, A. Ronge-Toloraya und H. Löffler-Stastka geben an, dass kein Interessenkonflikt besteht.

Für diesen Beitrag wurden von den Autoren keine Studien an Menschen oder Tieren durchgeführt. Für die aufgeführten Studien gelten die jeweils dort angegebenen ethischen Richtlinien.

Open Access. Dieser Artikel wird unter der Creative Commons Namensnennung 4.0 International Lizenz veröffentlicht, welche die Nutzung, Vervielfältigung Bearbeitung, Verbreitung und Wiedergabe in jeglichem Medium und Format erlaubt, sofern Sie den/die ursprünglichen Autor(en) und die Quelle ordnungsgemäß nennen, einen Link zur Creative Commons Lizenz beifügen und angeben, ob Änderungen vorgenommen wurden.

Die in diesem Artikel enthaltenen Bilder und sonstiges Drittmaterial unterliegen ebenfalls der genannten Creative Commons Lizenz, sofern sich aus der Abbildungslegende nichts anderes ergibt. Sofern das betreffende Material nicht unter der genannten Creative Commons Lizenz steht und die betreffende Handlung nicht nach gesetzlichen Vorschriften erlaubt ist, ist für die oben aufgeführten Weiterverwendungen des Materials die Einwilligung des jeweiligen Rechteinhabers einzuholen.

Weitere Details zur Lizenz entnehmen Sie bitte der Lizenzinformation auf http://creativecommons.org/ licenses/by/4.0/deed.de. 


\section{Literatur}

1. Hobsbawm E (1998) Das Zeitalter der Extreme Weltgeschichte des 20. Jahrhunderts. Deutscher Taschenbuchverlag

2. Bröckling U (2019) Das unternehmerische Selbst. Soziologie einer Subjektivierungsform. Suhrkamp Frankfurt, S 185

3. Coulter A (2002) The autonomous patient. Ending paternalism in medical care. The Nuffield Trust, London

4. Eibach U (1997) Vom Paternalismus zur Autonomie des Patienten? Medizinische Ethik im Spannungsfeld zwischen einer Ethik der Fürsorge und einer Ethik der Autonomie. ZMed Ethik 43(3):215-231

5. Ewert B (2013) Vom Patienten zum Konsumenten? Nutzerbeteiligung und Nutzeridentitäten im Gesundheitswesen. SpringerVS, Wiesbaden

6. Kloiber O (2000) Patienten sind keine Kunden. Dtsch Arztebl 97(5):A-229/B-181/C-169 (Abrufba unter https://www.aerzteblatt.de/archiv/21023/ Patienten-sind-keine-Kunden)

7. Freidson E (1979) Der Ärztestand. Berufs- und wissenschaftssoziologische Durchleuchtung einer Profession. Ferdinand Enke, Stuttgart

8. Pelikan JM, Griessler E, Grundböck A, Krajic K (1998) Das virtuelle Krankenhaus - Ausweg oder Königsweg für die Krankenversorgung der Zukunft? In: Pelikan JM, Stacher A, Grundböck A, Krajic K (Hrsg) Virtuelles Krankenhaus zu Hause - Entwicklung und Qualität von Ganzheitlicher Hauskrankenpflege: Theoretische Konzepte, gesundheitspolitischer Kontext und praktische Erfahrungen in Europa. Facultas, Wien, S 15-39 (p31)

9. Heldal F, Tjora A (2009) Making sense of patient expertise. Soc Theory Health 7:1-19. https://doi. org/10.1057/sth.2008.17 (p 17)

10. Kivits J (2004) Researching the 'Informed Patient' The case of online health information seekers. Inf Commun Soc 7(4):510-530

11. Prior L (2003) Belief, knowledge and expertise: the emergence of the lay expert in medical sociology. Sociol Health IIIn 25:41-57 (p53)

12. Vogd W (2006) Von der Organisation Krankenhaus zum Behandlungsnetzwerk? Untersuchungen zum Einfluss von Medizincontrolling am Beispiel einer internistischen Abteilung. Berl J Soziol 2006(4):97-119

13. Begenau J, Schubert C, Vogd W (2010) Einleitung: Die Arzt-Patient-Beziehung aus soziologischer Sicht. In: Die Arzt-Patient-Beziehung. Kohlhammer, Stuttgart

14. Lenartz N, Soellner R, Rudinger G (2016) Health Literacy. Entwicklung und Bedeutung einer Schlüsselkompetenz für gesundheitsgerechtes Leben. In: Jungbauer M et al (Hrsg) Handbuch Gesundheitssoziologie. Springer Reference Sozialwissenschaften. Springer, Wiesbaden https://doi. org/10.1007/978-3-658-06477-8_15-1

15. Siegrist J (2005) Medizinische Soziologie, 6. Aufl. Elsevier, München, S286

16. Esposito E (1993) Der Computer als Medium und Maschine.ZSoziol 22(5):338-354 (p 344)

17. Wu T, Jia X, Shi H, Niu J, Yin X, Xie J, Wang X (2021) Prevalence of mental health problems during the COVID-19 pandemic: a systematic review and meta-analysis. J Affect Disord 281:91-98. https:// doi.org/10.1016/j.jad.2020.11.117

18. Salari N, Khazaie H, Hosseinian-Far A, KhalediPaveh B, Kazeminia M, Mohammadi M, Shohaimi S Daneshkhah A, Eskandari S (2020) The prevalence of stress, anxiety and depression within front-line healthcare workers caring for COVID-19 patients: a systematic review and meta-regression. Hum Resour Health 18(1):100. https://doi.org/10.1186/ s12960-020-00544-1

19. The Lancet Psychiatry (2021) COVID-19 and mental health. Lancet Psychiatry 8(2):87. https://doi.org/ 10.1016/S2215-0366(21)00005-5

20. Mitscherlich Schönherr O (2021) Freiheit in Krisenzeiten. https://www.br.de/radio/bayern2/ sendungen/zum-sonntag/zum-sonntagmitscherlich-schoenherr-100.html (BR Bayern 2). Zugegriffen: 23.12.2021

21. DGGG (2020) Statement der DGGG. Pressemitteilung vom 24.4.2020. https://www.sozial.de/ schutz-ja-paternalismus-nein!.html. Zugegriffen: 23.12.2021

22. Simpson SG, Reid CC (2014) Therapeutic alliance in videoconferencing psychotherapy: a review. Aust J Rural Health 22(6):280-299

23. Eichenberg C (2020) Psychotherapie in derCoronakrise. Trendwende in der Online-Psychotherapie. Dtsch Arzteblatt PP 2020(6):255

24. Killgore WDS, Cloonan SA, Taylor EC, Anlap I, Dailey NS (2021) Increasing aggression during the COVID-19lockdowns.J AffectDisord Rep 5:100163. https://doi.org/10.1016/j.jadr.2021.100163

25. Berkowitz L (1989) Frustration-aggression hypothesis: examination and reformulation. Psycho Bull 106(1):59-73

26. Breuer J, Elson M (2017) Frustration-aggression theory. In: Sturmey P (Hrsg) The Wiley handbook of violenceand aggression. Wiley-Blackwell, Chichester, S 1-12 https://doi.org/10.1002/ 9781119057574.whbva040

27. Eichenberg C (2021) Onlinepsychotherapie in Zeiten der Coronapandemie. Psychotherapeut 13:1-8. https://doi.org/10.1007/s00278-020 00484-0

28. Leslie E, Wilson R (2020) Sheltering in place and domestic violence: evidence from calls for service during COVID-19. J Public Econ 189:104241. https://doi.org/10.1016/j.jpubeco.2020.104241

29. Lawson M Piel MH, Simon M (2020) Child maltreatment during the COVID-19 pandemic: consequences of parentaljobloss on psychological and physical abuse towards children. Child Abuse Negl 110(Pt 2):104709. https://doi.org/10.1016/j. chiabu.2020.104709

30. Sherman WF, Khadra HS, Kale NN, Wu VJ, Gladden PB, Lee OC (2021) How did the number and type of injuries in patients presenting to a regional level I trauma center change during the COVID-19 pandemic with a stay-at-home order? Clin Orthop Relat Res 479(2):266-275. https://doi. org/10.1097/CORR.0000000000001484

31. Löffler-Stastka H, Blueml V, Boes C (2010) Exploration of personality factors and their predictive impact on therapy utilization: the externalizing mode of functioning. Psychother Res 20(3):295-308. https://doi.org/10.1080/ 10503300903436710

32. Livesley WJ, Jang KL, Vernon PA (1998) Phenotypic and genetic structure of traits delineating personality disorder. Arch Gen Psychiatry 55(10):941-948

33. Clarkin JF, Yeomans F, Kernberg OF (2006) Psychotherapy for borderline personality. American Psychiatric Publishing, Washington, DC

34. Steiner J (1997) Problems of psychoanalytic technique: patient centered and analyst-centered interpretations. In: Schafer R(Hrsg) The contemporary Kleinians of London. International Universities Press, Madison, S372-392

35. Clarkin JF, Levy KN, Lenzenweger MF, Kernberg OF (2007) Evaluating three treatments for borderline personality disorder: a multiwave study. Am J Psychiatry 164(6):922-928

36. Datz F, Wong G, Löffler-Stastka H (2019) Interpretation and working through contemptuous facial micro-expressions benefits the patienttherapist relationship. Int J Environ Res Public Health 16(24):4901. https://doi.org/10.3390/ ijerph16244901

37. Löffler-Stastka H (2021) Kommunikativer Umgang mit Gewalt im Gesundheitswesen. Plenarvortrag MedUniWien

38. Wesuls R, Heinzmann T, Brinker L (2005) Professionelles Deeskalationsmanagement (ProDeMa). Praxisleitfaden zum Umgang mit Gewalt und Aggression in den Gesundheitsberufen, 4. Aufl.Unfallkasse Baden-Württemberg, Stuttgart (Abrufbar unter: https://www.gesundheitsdienstportal.de/ risiko-uebergriff/infoplus/5_4_3b.pdf)

39. Favrod J, Nguyen A, Tronche A-M, Blanc O, Dubreucq J, Chereau-Boudet I, Capdevielle D Llorca Pierre M (2019) Impact of positive emotion regulation training on negative symptoms and social functioning in schizophrenia: a field test. Front Psychiatry 10:532. https://doi.org/10.3389/ fpsyt.2019.00532

40. Vaskinn A, Andersson S, Østefjells T, Andreassen OA, Sundet K (2018) Emotion perception, nonsocial cognition and symptoms as predictors of theory of mind in schizophrenia. Compr Psychiatry 85:1-7. https://doi.org/10.1016/j.comppsych 2018.05.002

41. Norwood C, Moghaddam NG, Malins S, SabinFarrell R (2018) Working alliance and outcome effectiveness in videoconferencing psychotherapy: a systematic review and noninferiority metaanalysis. Clin Psychol Psychother 25(6):797-808 https://doi.org/10.1002/cpp.2315

Hinweis des Verlags. Der Verlag bleibt in Hinblick auf geografische Zuordnungen und Gebietsbezeich nungen in veröffentlichten Karten und Institutsadressen neutral. 